\title{
Investigation of a Novel Technique for Decline Curve Analysis in Comparison with the Conventional Models
}

\author{
Mehdi Bahari \\ Moghadam \\ Ahwaz Faculty of \\ Petroleum \\ Petroleum University \\ of \\ Technology, Iran
}

\author{
Alireza Rostami \\ Ahwaz Faculty of \\ Petroleum \\ Petroleum University \\ of \\ Technology, Iran
}

\author{
Edris Joonaki \\ Ahwaz Faculty of \\ Petroleum \\ Petroleum University \\ of \\ Technology, Iran
}

\author{
Payam \\ Mohammad Ali \\ Nejad \\ Ahwaz Faculty of \\ Petroleum \\ Petroleum University \\ of \\ Technology, Iran
}

\begin{abstract}
It has been proven that the common Arps empirical decline curve analysis (DCA) to be an extensively used tool for many years, however, it has much error in production forecasting. In the past, engineers forecasted the oil production rates without understanding of the reservoir engineering principals behind and without having the reservoir properties and operating conditions. These problems influences the quality of the production prediction by Arps model and decreases the validity of this technique. In addition, even by having those properties and conditions a reliable production prediction still cannot be promised without understanding of the theory that connects reservoir properties and operation to the production decline. Experience discovered that the production decline is completely complex that can't be handled just by a simple mathematical model such as Arp, so the lack of an efficient tool for DCA is still essential. This paper presents the simultaneous use of the conventional Arps method and ANNPSO as forecasting tools to find the optimum decline curve model. Production data from Ramin oilfield in Iran is analyzed by using these two techniques to forecast future the production performance. Arps decline curve and ANN-PSO models are compared, and the most appropriate approach is introduced in this paper. Consequently it is found out that ANN-PSO model gives a better results and can be used as a novel tool for DCA.
\end{abstract}

\section{GENERAL TERMS}

Modelling, Artificial Intelligence, Decline Curve Analysis, Petroleum Engineering

\section{Keywords}

Decline curve analysis (DCA), Arps model, ANN-PSO, Oil Production forecasting, Ramin Oilfield.

\section{INTRODUCTION}

Decline curve analysis is one of the most common approaches that is practical extensively for evaluation of oil and gas reserves and their properties. Generally there are three basic models of rate-time decline equations that are sorted as exponential, hyperbolic and harmonic equations based upon their b-values. Examination of the Arps equations shows the paramount significance of the decline exponent, $b$, that was investigated to be dependent on fluid properties and production conditions and determines the degree of curvature of the decline [1]. Arps proposed that the b-varies between zero and one, with no discussion of the possibility of $b>1$. However, there is no theoretical basis for limiting the exponent to values less than one. If there is a mechanism that maintains the reservoir pressure, like gas or water injection, an active water drive, or gas-cap drive, the production rate would mainly maintain constant and the decline tends toward zero. Since the decline in reservoir pressure is small, the production driving force remains large, and the decline in the producing rate is smaller correspondingly.

The main theoretical assumption for these decline equations are that the well is in boundary-dominated flow condition in a way that the skin factor, productivity index, drainage radius and flowing bottomhole pressure are constant. On top of that, in this method if we assume that the past reservoir behavior is conveyed and exerted with the same trend into the future of the well; the future of well behavior is followed up by the same past reservoir dynamics of the fluid.

Also it employs the mathematical equations to predict the production of declining and early producing fields respectively. It does not require any assumptions about the reservoir parameter. And it needs appropriate production data to permit the logical extrapolation.

The most popular types of decline curves which are used extensively are [2]: 1- Production Rate vs. Time, 2Cumulative Production vs. Time, 3- Production Rate vs. Cumulative Production.

Well production rate is the most accessible data of a producing well, so production decline-curve analysis has been carried out employing well production rate data. Commonly these data are existed on a daily or monthly format in formal repots, however, they are rarely evaluated on such a basis. Hence for production decline-curve analysis, some allocated measured well test rates has been used.

Constant-percentage decline (exponential decline) is the simplest type of decline curve model which can be handled both graphically and mathematically, therefore frequently expected production performance is extrapolated as a straight line on semi log scale. This is usually not true, and this is done regardless of the previous experiments which have revealed this scientific fact that hyperbolic decline is frequently the dominant decline model [3]. However, the mathematical or graphical analysis of hyperbolic model is hard to do in comparison with harmonic and exponential decline. Fetkovich [4] presented type curves combining the transient rate and the pseudo-steady state decline curves and derived single-phase 
flow from material balance and Darcy law. Lefkovits et al. [5] derived the exponential decline form for gravity drainage reservoirs by neglecting capillary pressure. Doublet et al. [6] constructed the theoretical basis for combining transient and boundary dominated production behavior for the pressure transient solution to the diffusivity equation.

The extensively used model for estimation of well ultimate recovery is to plot its production rate versus time or versus cumulative production, so by extrapolating semi-log straightline and intersecting with the economic scope of the well, it can be obtained.

Most publications on the subject are devoted to the development of the most realistic, general, and complicated hyperbolic decline. Conventional hyperbolic decline curve analysis is trial-and-error [7] or type curve matching [8] method. Other methods to decline curve analysis are less popular. Doung [9] manipulated rate and cumulative rate equations to gain a universal relation with three unknowns. He solved this equation by utilizing linear multiple regression. The limitations of this technique is that cumulative production is contained in determination of the production rate at each time step, which again includes a trial and error procedure or stepwise calculations to predict production.

A review of the existing literature on production decline curve analysis reveals that hardly any information is available on our proposed subject. This paper presents an intelligence approach to find the optimum decline curve model that was not reported in any research project before, and compare it with Arps decline curve model to introduce a more applicable technique for the petroleum industry.

\section{CASE STUDY}

Ramin oilfield is approximately $40 \mathrm{~km}$ long and $4 \mathrm{~km}$ wide which is located in $30 \mathrm{~km}$ northeast of Ahvaz and northwest of Maron reservoir. This oilfield contains Asmari and Bangestan reservoirs which include 4 producing wells of 8,9 , 14 and 15 in Asmari reservoir. Also it produces the light oil of Asmari reservoir. From geological point of view, this reservoir is an elongated anticline which has two axes in north and southwest strikes. 8, 9, 14 and 15, which are studied in this paper, are located in eastern sector (pay zone) of Asmari reservoir.

\section{METHODOLOGIES}

\subsection{Basic Theory of Decline Curve Analysis Methods}

Decline curve analysis is disputably the most prevalently utilized method for prediction of reserves in oil reservoirs, and basic theory and applications are argued in a number of reference papers. At the most fundamental level, decline curve analysis contains fitting an empirical model to the trend in production decline from a well's history, and projecting the trend into the future to evaluate the well's economic life and predicting its cumulative production.

Cartesian or semi-logarithmic plots of flow rate versus time and/or rate versus cumulative production are usually employed.

Rate versus time plots can be employed for economic predicting, but rate versus cumulative production plots are also utilized to clarify production to an economic limit. Two key points involve:

1- To employ any form of decline curve analysis, the producing wells must show production decline. If only initial production or peak production rate data are accessible, it may be possible to establish a correlation between these and EUR in analogous wells, thereby justifying EUR predicting by utilizing a suitable type curve.

2- The selected approach of curve fitting or production decline equation has a significant influence on rate forecasting, and the prediction of the EUR in a well accordingly. Even basic approaches give tremendously different EUR estimates at a given abandonment time or rate [10].

\subsection{ARPS Model}

The simplest decline curve analysis approaches are those presented by Arps. It is applicable to outline these, since they are still commonly employed, and the theory constitutes the basis for some of the more complex techniques. The Arps decline model is a base upon the empirical observation that the loss ratio (the rate of change of the reciprocal of the instantaneous decline rate) is constant with time [10]. In the most cases production will decline at a decreasing rate. The rate of decline is expressed by [11]:

$$
D=-\frac{1}{q} \frac{d q}{d t}
$$

The decline rate is also approximated by a power function:

$$
D=a q^{b}
$$

Substitution Eq. 2 into Eq. 1 yields a differential equation, which could be solved for production rate, q.

$$
a q^{b}=-\frac{1}{q} \frac{d q}{d t}
$$

The equation is appropriate for the Pseudo-Steady State flow condition. Nearly all conventional decline curve analysis is based upon the empirical rate-time equation given by Arps as [2]:

$$
\frac{q(t)}{q_{i}}=\frac{1}{\left[1+b D_{i} t\right]^{\frac{1}{b}}}
$$

For $b=0$, we can obtain the exponential decline equation for Eq. 4:

$$
\frac{q(t)}{q_{i}}=\frac{1}{e^{D_{i}}}
$$

For $\mathrm{b}=1$, referred to as harmonic decline curve, we have:

$$
\frac{q(t)}{q_{i}}=\frac{1}{\left[1+D_{i} t\right]}
$$

The results are plotted a developed for values of $\mathrm{b}$ between 0 and 1 in 0.1 increments as a set of log-log type curves for a unit solution $(\mathrm{Di}=1)$ in terms of a decline curve dimensionless rate, which is presented in figure 1 ;

$$
q_{D d}=\frac{q(t)}{q_{i}}
$$

And a decline curve dimensionless time: 


$$
t_{D d}=D_{i} t
$$

Table 1 presents a brief review of Arps decline curve analysis.

\subsection{PARTICLE SWARM OPTIMIZATION (PSO)}

Particle swarm optimization (PSO) algorithm, which fundamentally presented by Kennedy and Eberhart, proved that has a better execution in comparison with other population based methods of optimization. It is a stochastic optimization method which inspired from social treatment of birds and fishes. PSO successfully applied in different fields , which is not only compare with other approaches of this region including Fuzzy computation and Chaos theory but also with other stochastic methods like genetic algorithm (GA) has a better act with shorter time and less error. Another reason that makes PSO an interesting technique of optimization is the small number of parameters that employed in it. Regardless of having a simple structure, implementing PSO is a comfortable process. PSO models the exploration of parameter space by moving agents through the investigating space with following the recent optimum particles. The successful history of each particle influences its future movement. Every particle in this algorithm is a nominee for the answer. In this algorithm particles has an initial position that selected randomly then a local best (lb) is determined for every particle during each movement that is depended on that special one and for group of particles a global best $(\mathrm{gb})$ is determined which is a part of the entire group. Every particle's position updated by a vector that has a linear relation with local best and global best, through each iteration as below:

$$
\mathrm{X}_{2}=\mathrm{X}_{1}+\mathrm{V}
$$

$$
\mathrm{V}=\mathrm{C}_{1} \mathrm{r}_{1}\left(\mathrm{X}_{\mathrm{lb}}-\mathrm{X}_{1}\right)+\mathrm{C}_{2} \mathrm{r}_{2}\left(\mathrm{X}_{\mathrm{gb}}-\mathrm{X}_{1}\right)
$$

The first position of each particle is its initial local best and the best initial position among set of particles is their initial global best. Local best is the best position (having the best cost) of particle that had until that moment. And global best is the best position among all particles until that moment. The algorithm continues until the convergence criterion such as minimum error or maximum iteration is satisfied.

\subsection{ARTIFICIAL NEURAL NETWORKS (ANNs)}

Artificial neural networks (ANNs) are parallel information processing approaches that utilized to define complex and nonlinear relationship and employs number of input-output training patterns from the experimental data. Finding a nonlinear algorithm between inputs and outputs is obtained by natural ability of ANNs. They are made of nodes or neurons, number of simple computing components, which utilized to form respectively an input layer, one or more hidden layers and an output layer [12]. Feed-Forward neural network architecture, network structure in which the information or signals will generate only in one direction, from input to output, is the most common neural network architecture. The number of inputs in the process is the same as the number of nodes in the feed forward neural network input layer, whereas the number of output nodes is equal to the number of process output [13]. Every nonlinear continuous function can be approximated by three-layered feed-forward neural network with back propagation algorithm to an arbitrary precision [14].
The network is trained by carrying out optimization of weights for each neurons interconnection and bias term until the estimated values at the output layer neurons are as close as possible to the actual outputs. The mean squared error of the network (MSE) is determined by:

$$
M S E=\frac{\sum_{i=1}^{N}\left(Z_{i}^{\text {pred }}-Z_{i}^{\exp }\right)^{2}}{N}
$$

The data are split into two sets, a training data set and a testing data set. Only training data are employed for producing model. The testing data are utilized to test the model and proves its accuracy. Finding an optimum set of weights is the objective of training a network. When the number of available data is smaller than the number of weights, the error in fitting the non-trained data reduces at first, but then rises as the network becomes over trained. In contrast, when the number of data is larger than the number of weights, the over fitting problem is not very significant.

\section{RESULTS AND DISCUSSIONS}

In this study, a hybrid version of Artificial Neural Network and particle swarm optimization (ANN-PSO) method was employed to predict the oil production rate. PSO is used as neural network weight initialization algorithm and the Mean Square Error (MSE) utilized as a cost function for this algorithm. The purpose in the proposed algorithm is minimizing of this cost function. In these simulations, the number of swarms is 70 parameters $\mathrm{C} 1$ and $\mathrm{C} 2$ are 2.02 and 2 respectively. The number of training and testing data is 150 and 40 respectively. The simulation performance of the ANNPSO model was evaluated on the basis of mean square error (MSE), root mean square error (RMSE) and efficiency coefficient R2. Tables 2, 3, 4 and 5 give the MSE, RMSE and R2 values for ANN-PSO method based upon different number of neurons in hidden layer, and the most accurate one is selected as the base of our comparison. In each well the best model is found to be fitted on the production history of individual wells. In accordance to the figures of Arps model and those of ANN-PSO modeling, it is clear that in all cases ANN-PSO modeling gives a higher value for $\mathrm{R}^{2}$ (nearer to unity) and consequently less error are taken into consideration by this method of analysis. Based on the figure 2 and 4 for wells 8 and 14, when logarithm of flow rate vs. cumulative production becomes linear, harmonic model will happen. According to the figure 3, the diagnosing (forecasting) model for well 9 is exponential because of the linear relationship in this diagram. Also the $\mathrm{R}^{2}$-value of this well is the minimum one among other well models. Figure 5 illustrates a general hyperbolic model for well 15 which occurs when no linear relation observed. Final ANN-PSO models presented higher R2 values for the training and testing data sets of each well than the Arp well models. Output of ANN-PSO model versus real output for training and testing data sets of wells $8,9,14$ and 15 are presented in figures $6,7,8,9,10,11,12$ and 13 respectively.

\section{CONCLUSION}

Application of artificial intelligence as novel tool in DCA was investigated. Arps decline curve and ANN-PSO models are compared, and the most appropriate approach is introduced for each well in Ramin oilfield. Finally following conclusions are made:

1- A hybrid version of ANN-PSO presents an appropriate result, and it can be recognized as a novel tool and a new 
technique for decline curve analysis rather than common model of Arp.

2- It is observed that when we are dealing with exponential model which is highly optimistic, both Arp and ANN-PSO will results in a low value for R2 and consequently a high error would happen.

3- Based on the results of this case study, it is concluded that among different types of Arps model, the harmonic decline is the most common type.

\section{ACKNOWLEDGMENT}

The authors are so grateful to The Petroleum Engineering Department in Ahwaz Faculty of Petroleum Engineering, and the National Iranian Oil Company for providing data.

\section{APPENDIX A: STATISTICAL} FORMULA

Coefficient of determination:

$$
R^{2}=1-\frac{\sum_{i=1}^{N}\left(Z_{i}^{\text {pred }}-Z_{i}^{\text {exp }}\right)^{2}}{\sum_{i=1}^{N}\left(Z_{i}^{\text {pred }}-\operatorname{average}\left(Z_{i}^{\exp }\right)\right)^{2}}
$$

Mean squared error:

$$
M S E=\frac{\sum_{i=1}^{N}\left(Z_{i}^{\text {pred }}-Z_{i}^{\exp }\right)^{2}}{N}
$$

Average mean squared error:

$$
R M S E=\left(\frac{\sum_{i=1}^{N}\left(Z_{i}^{\text {pred }}-Z_{i}^{\exp }\right)^{2}}{N}\right)^{\frac{1}{2}}
$$

\section{NOMENCLATURE}

b decline exponent

$\mathrm{D}_{\mathrm{i}} \quad$ relative decline rate, dimensionless

q current surface oil rate of flow, STBD

$\mathrm{q}_{\mathrm{i}} \quad$ initial surface oil rate of flow, STBD

$q(t) \quad$ current oil rate of flow, STBD

$\mathrm{q}_{\mathrm{D}} \quad$ dimensionless rate

$\mathrm{q}_{\mathrm{Dd}} \quad$ decline-curve dimensionless rate

$\mathrm{t}$ flow time, hour

$t_{D} \quad$ dimensionless time

$t_{\mathrm{Dd}} \quad$ decline-curve dimensionless time

$\mathrm{C}_{1} \quad$ first constant of PSO method

$\mathrm{C}_{2} \quad$ second constant of PSO method

$\mathrm{r}_{1} \quad$ first random coefficient of PSO method

$\mathrm{r}_{2} \quad$ second random coefficient of PSO method

$\mathrm{N}$ number of training samples

$Z_{i}^{\text {pred }} \quad$ estimated output

$Z_{i}^{e x p} \quad$ actual output 
Table 1. A brief review of Arps decline curve analysis (Gentry, R.W., 1996)

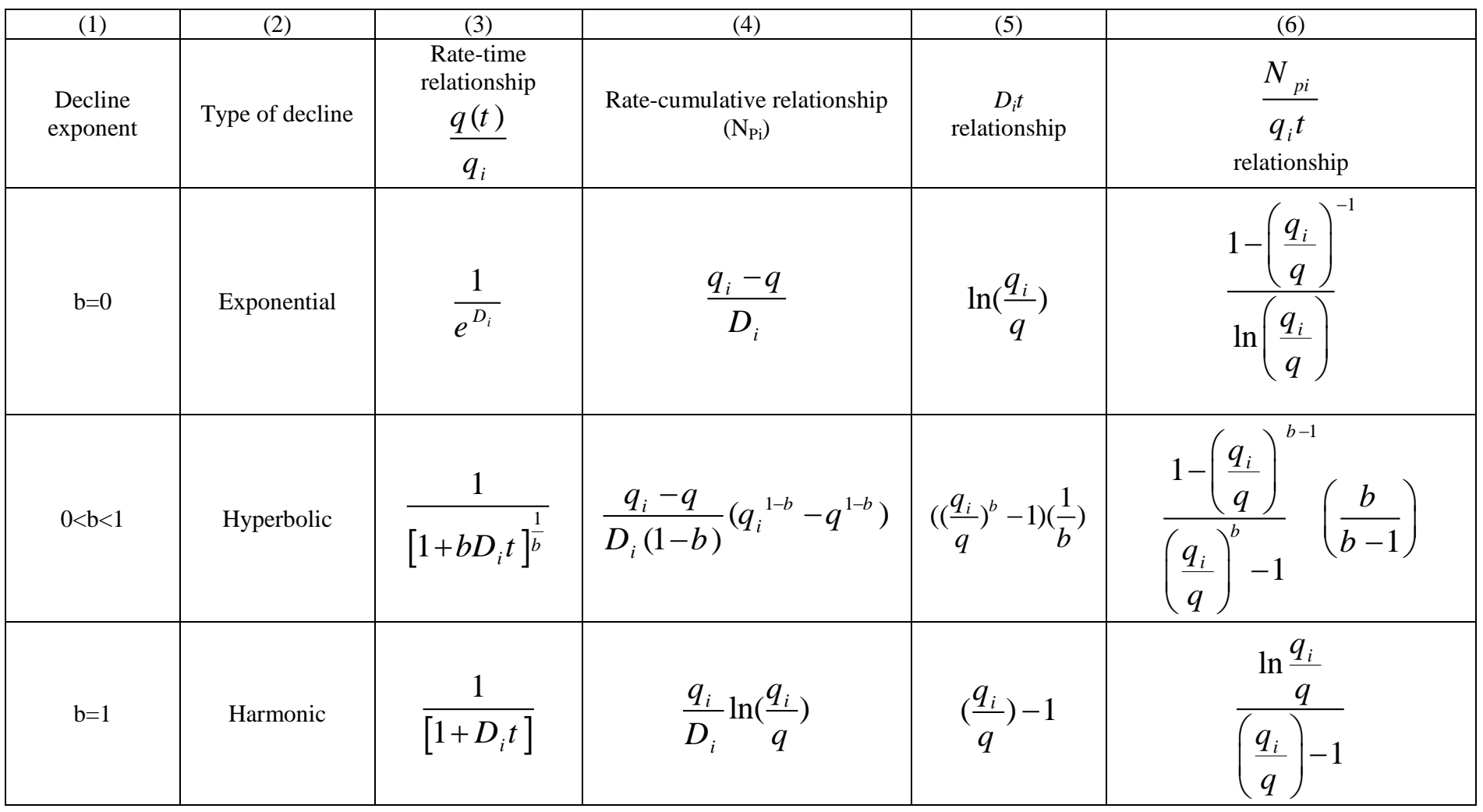

Table 2. MSE, RMSE and R2 of ANN-PSO modeling for well 8

\begin{tabular}{|c|c|c|c|c|c|c|c|}
\hline \multirow{2}{*}{$\begin{array}{c}\text { N o } \\
\text { Runs }\end{array}$} & \multirow{2}{*}{ neurons } & \multicolumn{3}{|c|}{ Training } & \multicolumn{2}{c|}{ Testing } \\
\cline { 2 - 7 } & & $\mathrm{R}^{2}$ & MSE & RMSE & R $^{2}$ & MSE & $\mathbf{0 . 0 4 5 3}$ \\
\hline $\mathbf{1}$ & $\mathbf{2}$ & $\mathbf{0 . 5 1 8}$ & $\mathbf{0 . 0 5 0 5}$ & $\mathbf{0 . 2 2 4 7}$ & $\mathbf{0 . 6 1 5 6}$ & 0.2129 \\
\hline 2 & 3 & 0.4901 & 0.0480 & 0.2191 & 0.6099 & 0.0372 \\
\hline 3 & 4 & 0.5253 & 0.0492 & 0.2218 & 0.3866 & 0.0535 \\
\hline 4 & 5 & 0.5191 & 0.0482 & 0.2195 & 0.5314 & 0.0291 \\
\hline 5 & 6 & 0.5893 & 0.0536 & 0.2315 & 0.2216 & 0.125 & 0.1707 \\
\hline 6 & 7 & 0.527 & 0.0511 & 0.2261 & 0.1614 & 0.3533 \\
\hline 7 & 8 & 0.5054 & 0.0370 & 0.1922 & 0.5842 & 0.0376 & 0.2441 \\
\hline 8 & 9 & 0.4999 & 0.0409 & 0.2022 & 0.5866 & 0.194 \\
\hline
\end{tabular}

Table 3. MSE, RMSE and R2 of ANN-PSO modeling for well 9

\begin{tabular}{|c|c|c|c|c|c|c|c|}
\hline \multirow{2}{*}{$\begin{array}{c}\text { N o } \\
\text { Runs }\end{array}$} & neurons & \multicolumn{3}{|c|}{ Training } & \multicolumn{3}{c|}{ Testing } \\
\cline { 3 - 7 } & & $\mathrm{R}^{2}$ & MSE & RMSE & $\mathrm{R}^{2}$ & MSE & RMSE \\
\hline 1 & 2 & 0.4997 & 0.0500 & 0.2238 & 0.5026 & 0.0522 & 0.2284 \\
\hline 2 & 3 & 0.5113 & 0.0488 & 0.2209 & 0.4781 & 0.0564 & 0.2376 \\
\hline 3 & 4 & 0.5211 & 0.0499 & 0.2235 & 0.5074 & 0.0464 & 0.2155 \\
\hline 4 & 5 & 0.5038 & 0.0547 & 0.2337 & 0.4843 & 0.0538 & 0.2319 \\
\hline $\mathbf{5}$ & $\mathbf{6}$ & $\mathbf{0 . 5 0 3 4}$ & $\mathbf{0 . 0 4 9 0}$ & $\mathbf{0 . 2 2 1 3}$ & $\mathbf{0 . 5 9 0 3}$ & $\mathbf{0 . 0 4 1 7}$ & $\mathbf{0 . 2 0 4 3}$ \\
\hline 6 & 7 & 0.5176 & 0.0642 & 0.2534 & 0.4372 & 0.0665 & 0.2579 \\
\hline 7 & 8 & 0.5048 & 0.0449 & 0.2118 & 0.5299 & 0.0488 & 0.2209 \\
\hline 8 & 9 & 0.5332 & 0.0476 & 0.2182 & 0.4675 & 0.0695 & 0.2636 \\
\hline
\end{tabular}


Table 4. MSE, RMSE and R2 of ANN-PSO modeling for well 14

\begin{tabular}{|c|c|c|c|c|c|c|c|}
\hline \multirow{2}{*}{$\begin{array}{c}\text { N o } \\
\text { Runs }\end{array}$} & \multirow{2}{*}{ neurons } & \multicolumn{3}{|c|}{ Training } & \multicolumn{3}{c|}{ Testing } \\
\cline { 3 - 8 } & & $\mathrm{R}^{2}$ & MSE & RMSE & $\mathrm{R}^{2}$ & MSE & RMSE \\
\hline 1 & 2 & 0.735 & 0.0497 & 0.2229 & 0.8057 & 0.0562 & 0.237 \\
\hline $\mathbf{2}$ & $\mathbf{3}$ & $\mathbf{0 . 7 5 0 3}$ & $\mathbf{0 . 0 4 9 6}$ & $\mathbf{0 . 2 2 2 8}$ & $\mathbf{0 . 8 5 2 1}$ & $\mathbf{0 . 0 1 9 9}$ & $\mathbf{0 . 1 4 0 9}$ \\
\hline 3 & 4 & 0.763 & 0.0530 & 0.2303 & 0.7282 & 0.0392 & 0.1981 \\
\hline 4 & 5 & 0.75 & 0.0499 & 0.2235 & 0.7981 & 0.0385 & 0.1962 \\
\hline 5 & 6 & 0.787 & 0.0419 & 0.2046 & 0.6647 & 0.0725 & 0.2692 \\
\hline 6 & 7 & 0.7713 & 0.0475 & 0.218 & 0.7236 & 0.0506 & 0.2249 \\
\hline 7 & 8 & 0.7903 & 0.0503 & 0.2243 & 0.6312 & 0.0808 & 0.2842 \\
\hline 8 & 9 & 0.7405 & 0.0544 & 0.2332 & 0.828 & 0.0517 & 0.2275 \\
\hline
\end{tabular}

Table 5. MSE, RMSE and R2 of ANN-PSO modeling for well 15

\begin{tabular}{|c|c|c|c|c|c|c|c|}
\hline \multirow{2}{*}{$\begin{array}{c}\text { N o } \\
\text { Runs }\end{array}$} & neurons & \multicolumn{3}{|c|}{ Training } & \multicolumn{3}{c|}{ Testing } \\
\cline { 3 - 7 } & & $\mathrm{R}^{2}$ & MSE & RMSE & $\mathrm{R}^{2}$ & MSE & RMSE \\
\hline 1 & 2 & 0.7512 & 0.0396 & 0.199 & 0.8181 & 0.0292 & 0.1709 \\
\hline 2 & 3 & 0.8322 & 0.0377 & 0.1942 & 0.8389 & 0.0330 & 0.1818 \\
\hline 3 & 4 & 0.8307 & 0.0361 & 0.1901 & 0.8613 & 0.0319 & 0.1785 \\
\hline 4 & 5 & 0.8827 & 0.0312 & 0.1767 & 0.893 & 0.0286 & 0.1691 \\
\hline 5 & 6 & 0.828 & 0.0350 & 0.1872 & 0.8509 & 0.0353 & 0.188 \\
\hline 6 & 7 & 0.9022 & 0.0270 & 0.1645 & 0.8145 & 0.0425 & 0.2061 \\
\hline 7 & 8 & 0.8553 & 0.0380 & 0.1949 & 0.8554 & 0.0325 & 0.1804 \\
\hline $\mathbf{8}$ & $\mathbf{9}$ & $\mathbf{0 . 9 1 8 1}$ & $\mathbf{0 . 0 2 5 0}$ & $\mathbf{0 . 1 5 8 3}$ & $\mathbf{0 . 8 8 7 7}$ & $\mathbf{0 . 0 2 8 0}$ & $\mathbf{0 . 1 6 7 4}$ \\
\hline
\end{tabular}

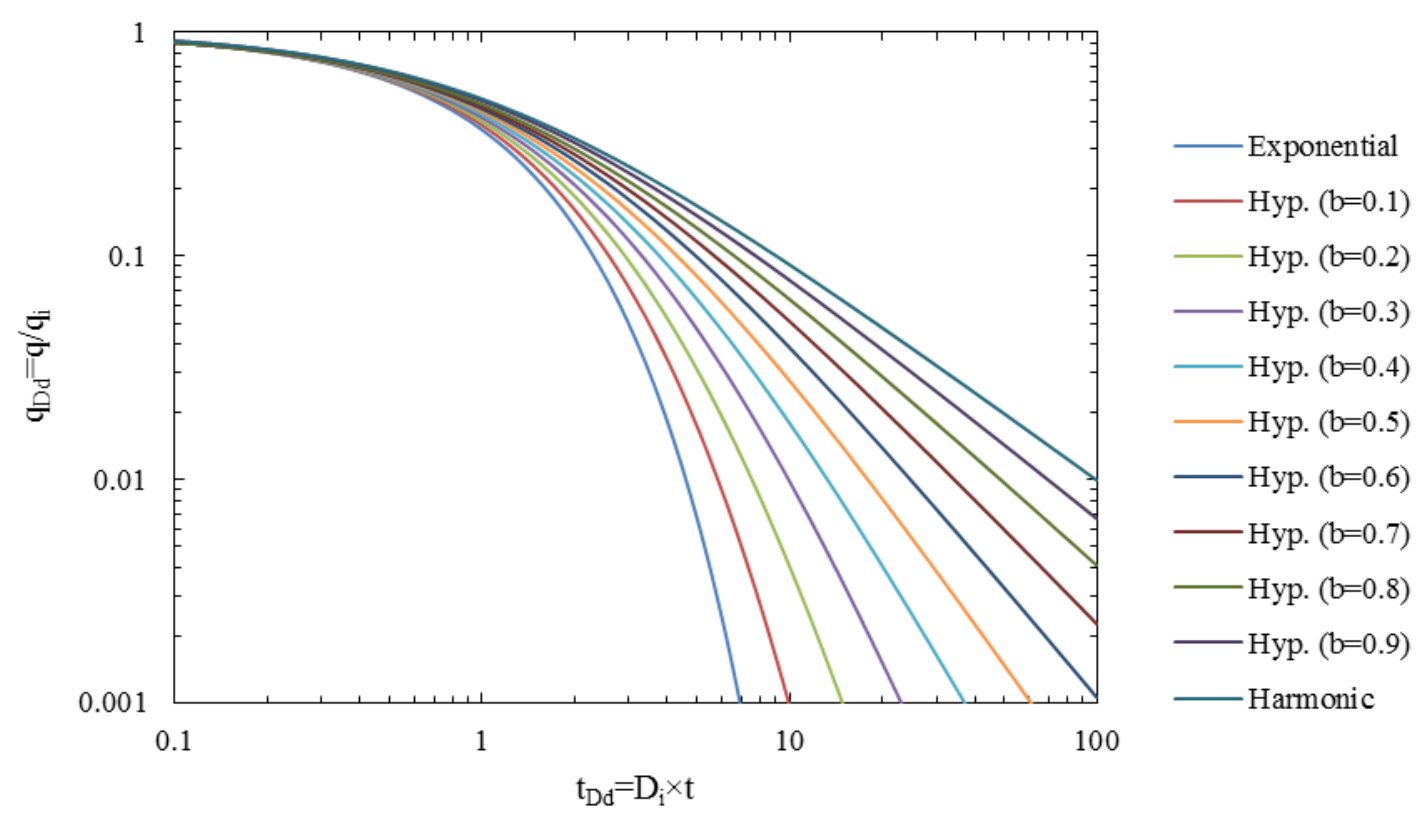

Figure 1. Type Curves for Arps Empirical Rate-Time Decline Equations in a log-log scale. 


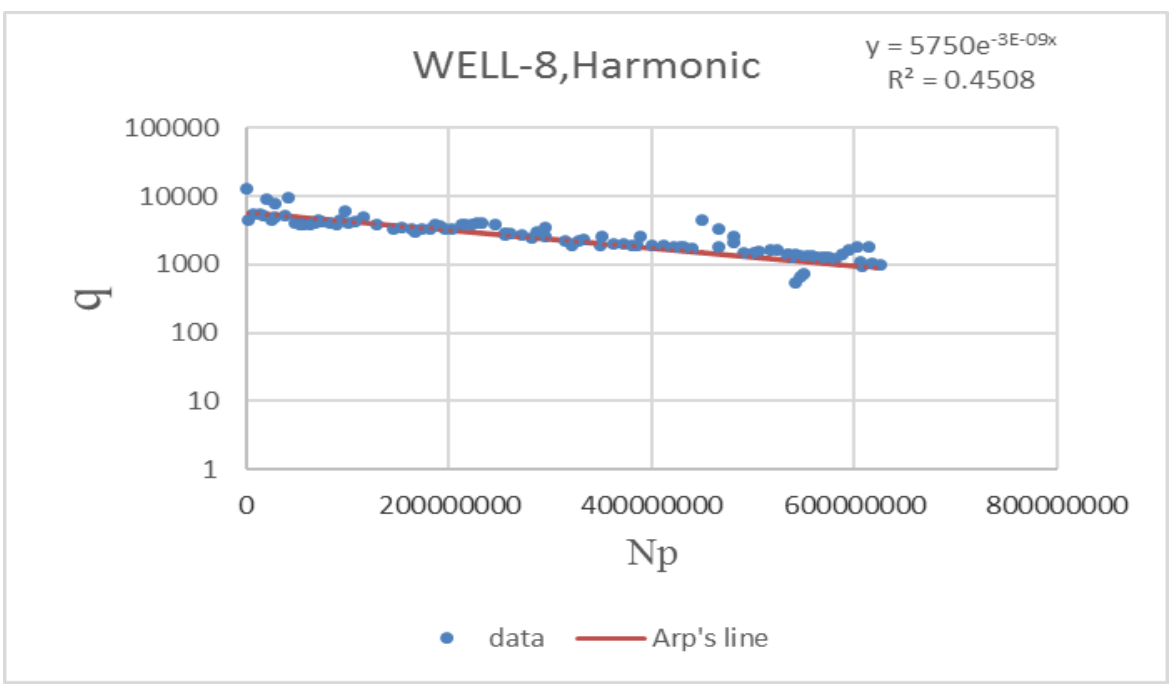

Figure 2. q vs. Np in a semilog diagram showing a harmonic decline for well 8 .

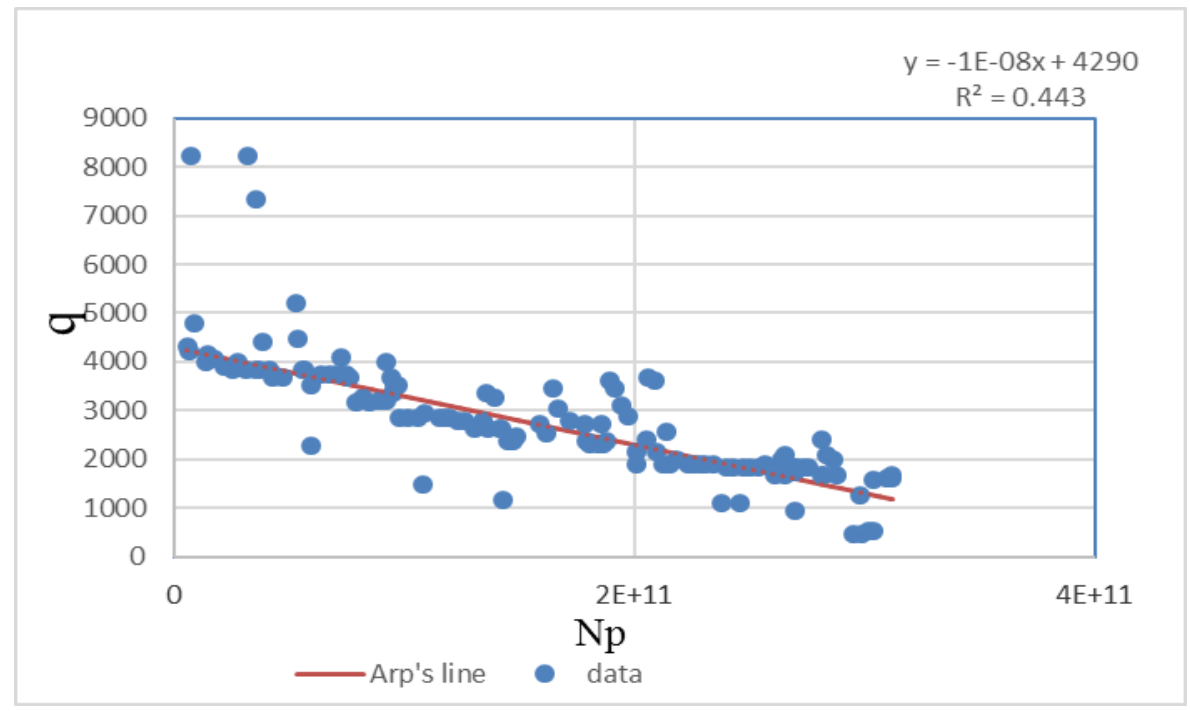

Figure 3: q vs. Np in a Cartesian diagram showing an exponential decline for well 9.

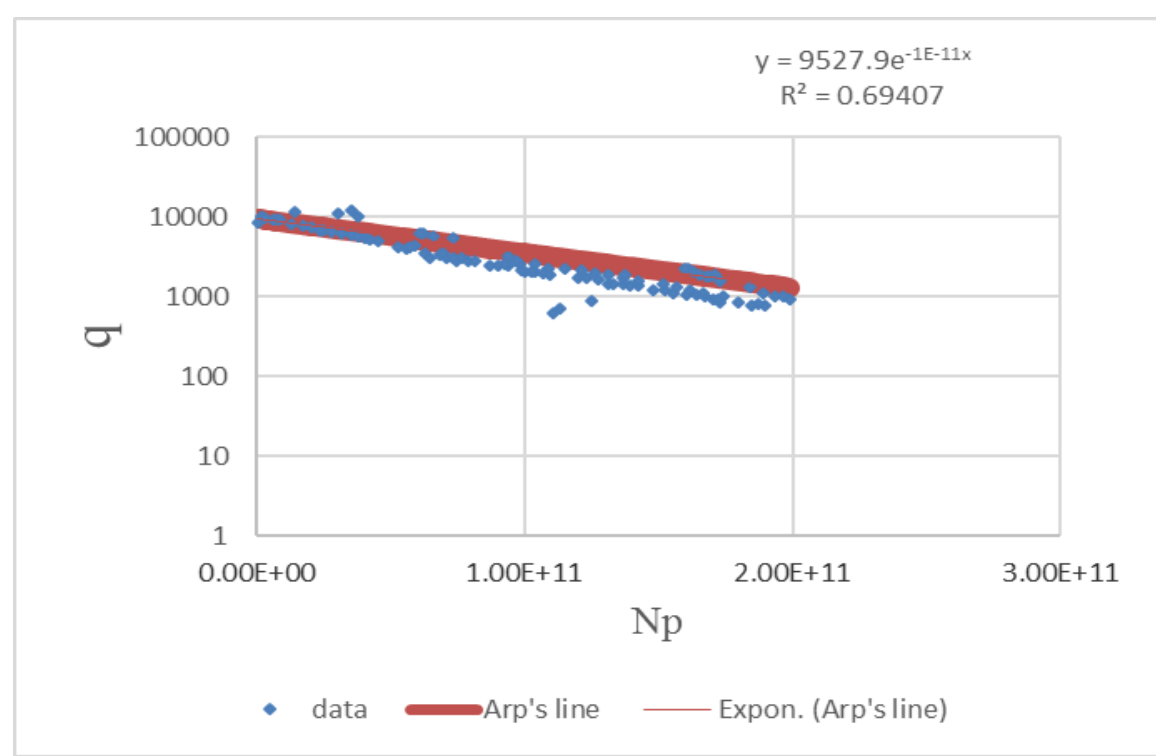

Figure 4. q vs. Np in a semilog diagram showing a harmonic decline for well 14. 


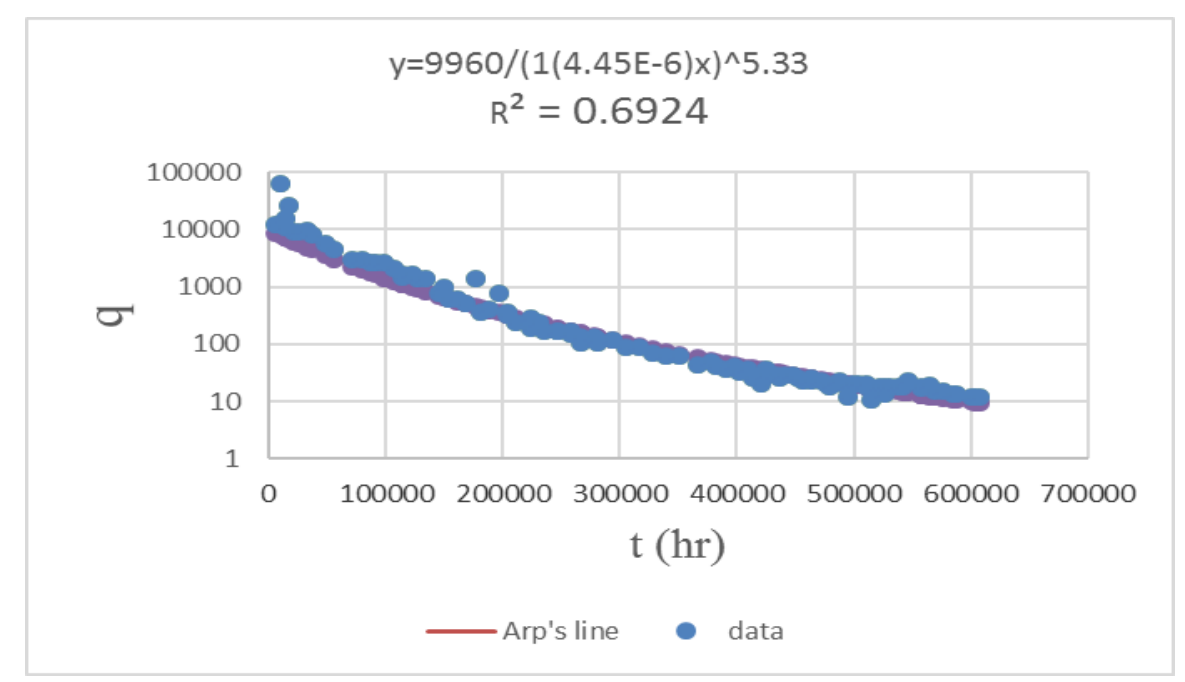

Figure 5. q vs. t (hr) in a semilog diagram showing a hyperbolic decline for well 15.

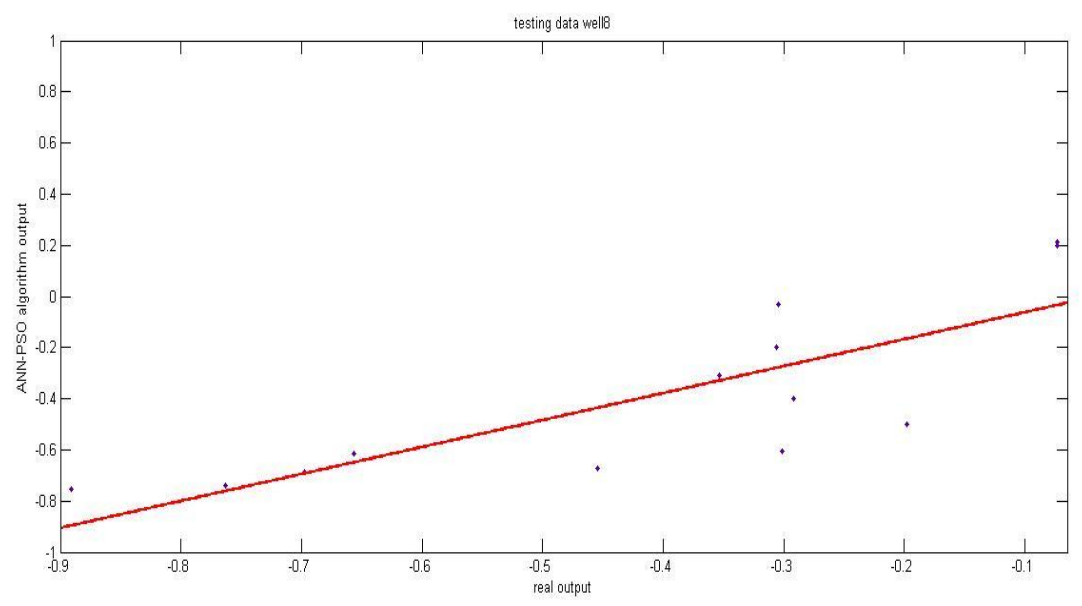

Figure 6. ANN-PSO output vs. real output for testing data set (Well 8)

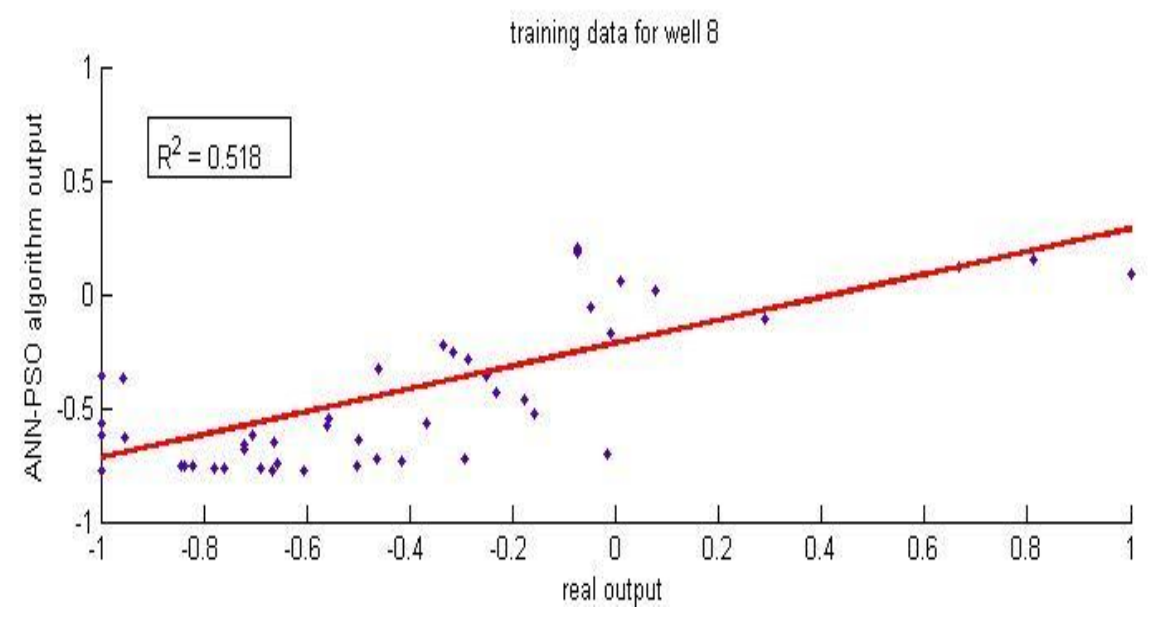

Figure 7. ANN-PSO output vs. real output for training data set (Well 8). 


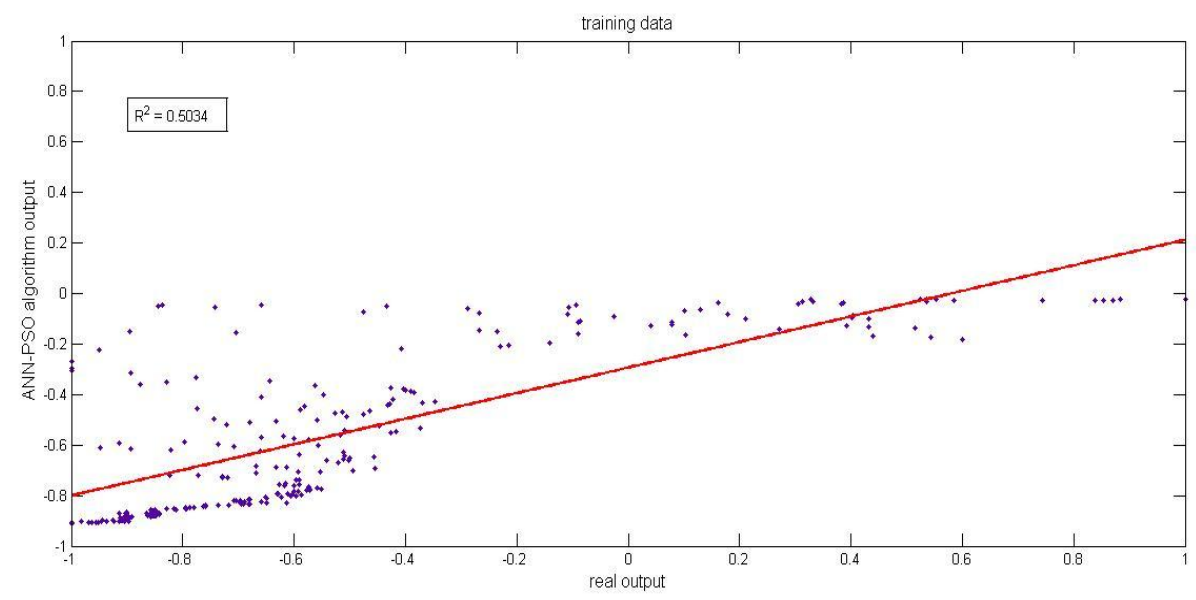

Figure8. ANN-PSO output vs. real output for testing data set (Well 9).

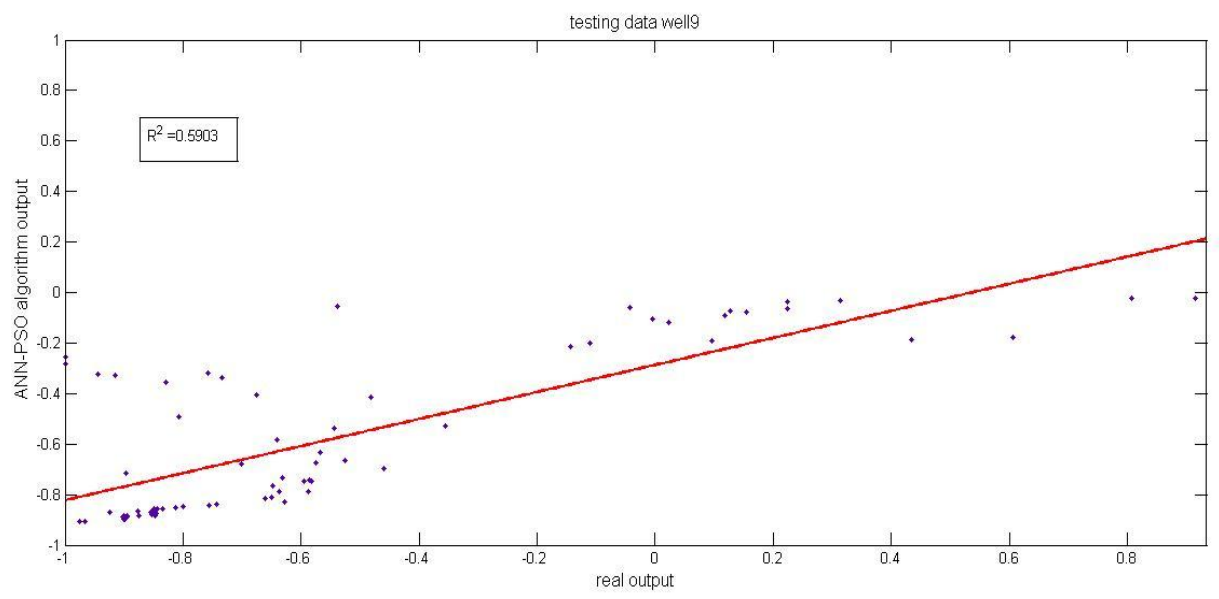

Figure 9. ANN-PSO output vs. real output for training data set (Well 9).

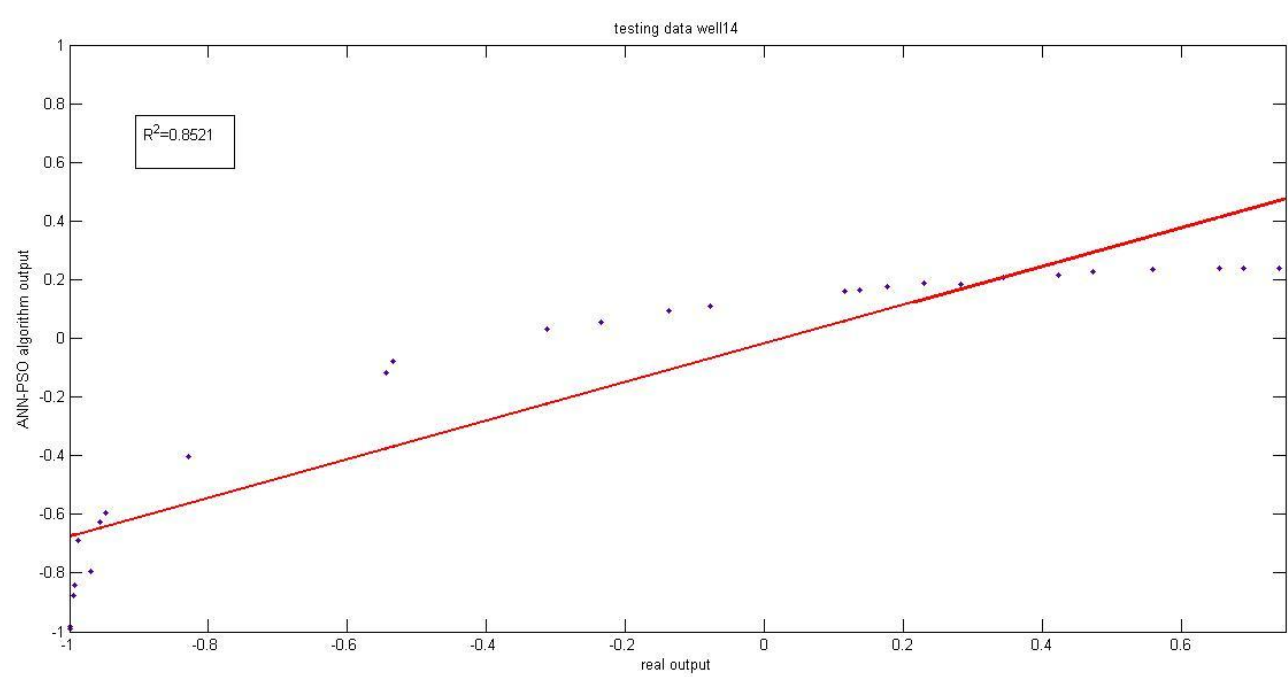

Figure 10. ANN-PSO output vs. real output for testing data set (Well 14). 


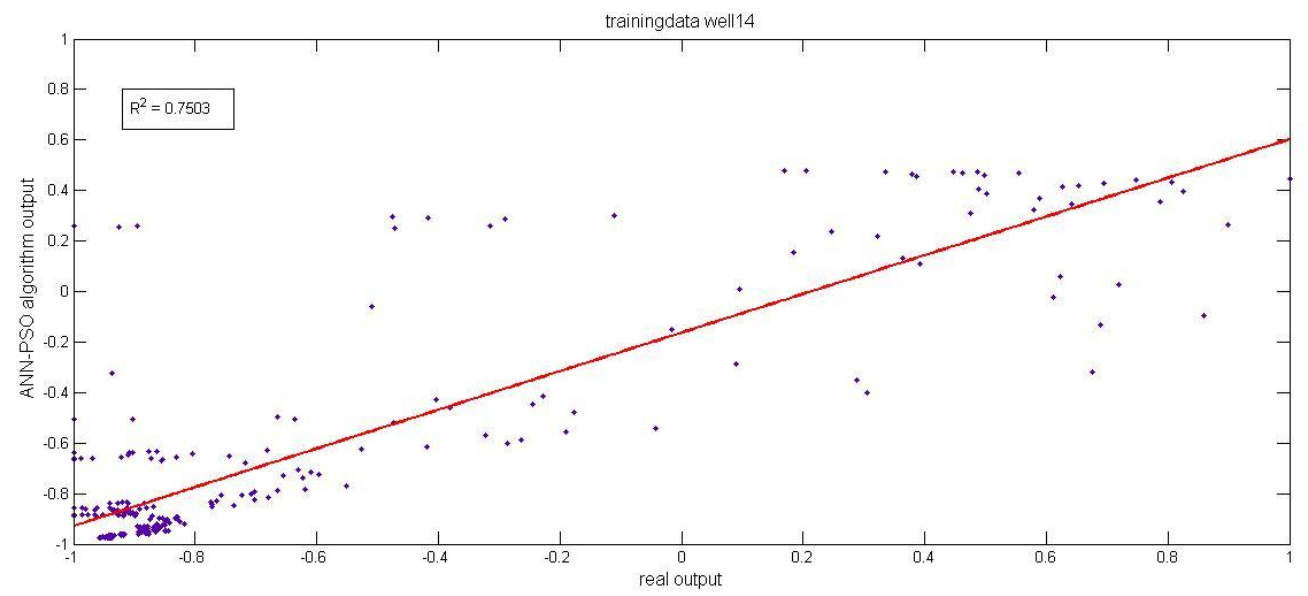

Figure 11. ANN-PSO output vs. real output for training data set (Well 14).

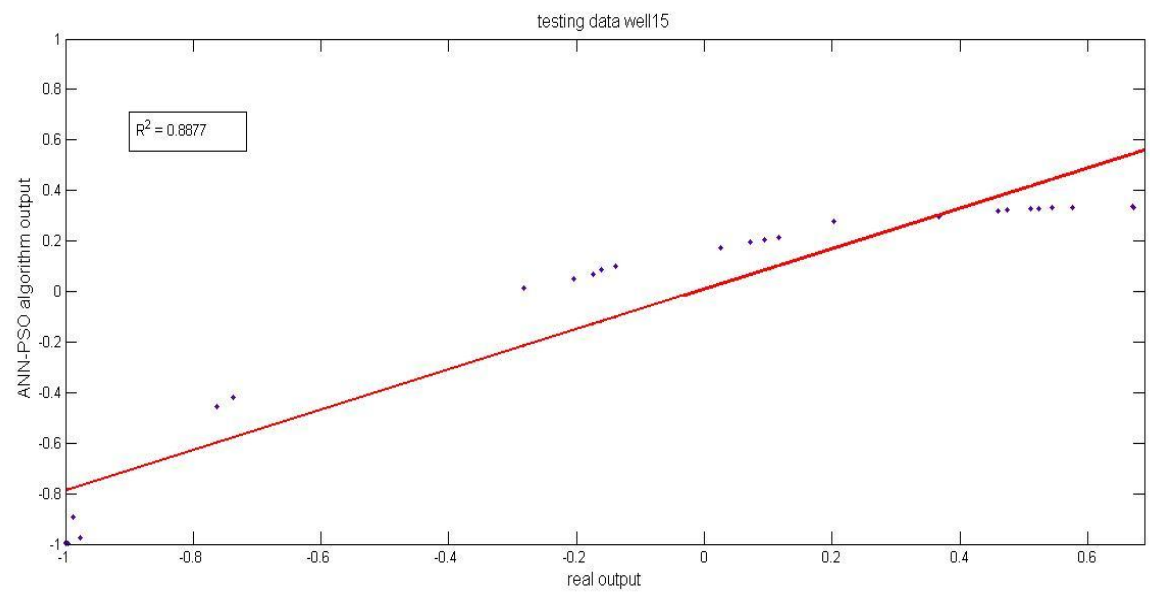

Figure 12. ANN-PSO output vs. real output for testing data set (Well 15).

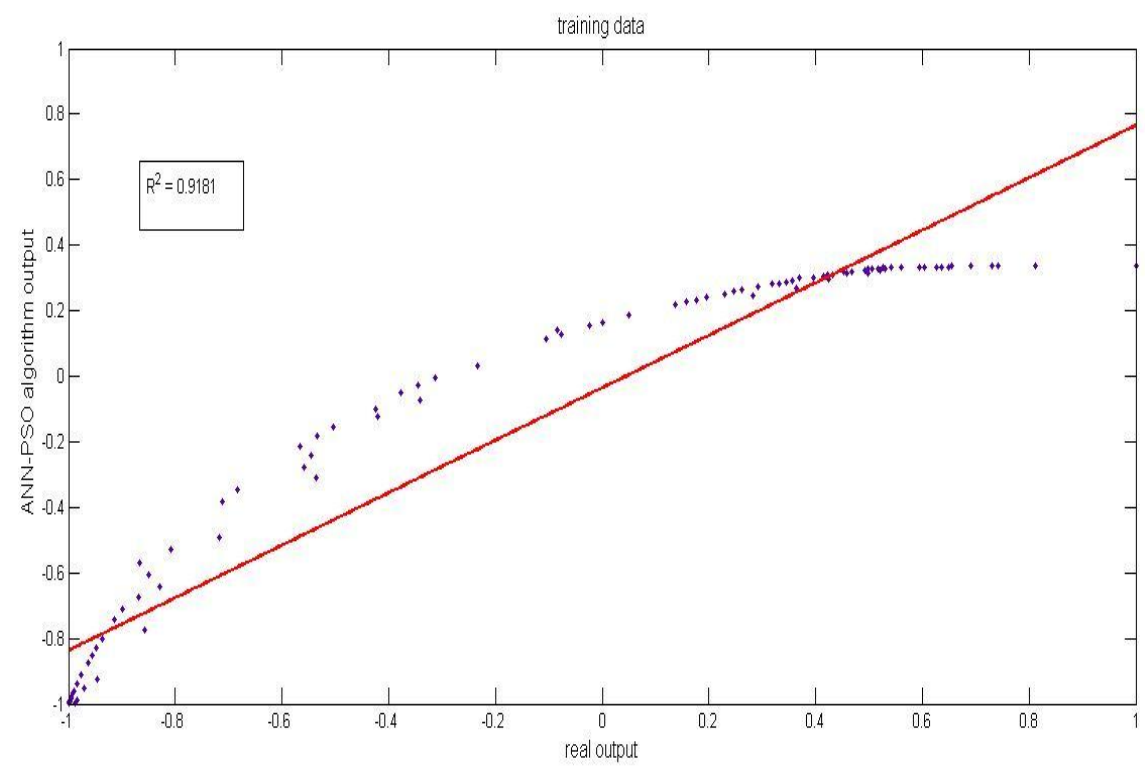

Figure 13. ANN-PSO output vs. real output for training data set (Well 15) 


\section{REFERENCES}

[1] Arps, J.J., 1945, "Analysis of Decline Curves," Trans. AIME, 228-247.

[2] Wang, J. 2008, "Decline Curve Analysis of Trattnach Oil Field", pp.6-10, $9^{\text {th }}$, M.Sc. Thesis.

[3] Gentry, R.W., 1996, "Decline Curve Analysis", pp.1, SPE-AIME, SPE-3356, Edinger, Inc.

[4] Fetkovich, M. J. 1980, June, "Decline Curve Analysis Using Type Curves," Journal of Petroleum Technology, Vol. 32, No. 6: 1065-77.

[5] Lefkovits, N.Cand, Matthews, C.S., 1958, "Application of Decline Curves to Gravity-Drainage Reservoir in the Stripper Stage," Trans. AIME, 213-277.

[6] Doublet, L. E., Pande, P.K., McCollum, T. J., and Blasingame, T. A. 1994. Decline Curve Analysis Using Type Curves Analysis of Oil Well Production Data Using Material Balance Time: Application to Field Cases, paper SPE 28688 presented at the Petroleum Conference and Exhibition of Mexico held in Veracruz, Mexico

[7] Spivey, J.P., 1986, "A new Algorithm for Decline Curve Fitting," SPE 15293. Symposium on Petroleum Industry Application of Microcomputers of SPE, Silver Creek, CO.
[8] Fetkovich, M.J., 1973, "Decline Curve Analysis Using Type Curves," SPE 4629, AIME 48 Annual Fall Meeting, Las Vegas.

[9] Doung, A. N., 1989, "A new Approach for DeclineCurve Analysis," SPE 18859, SPE Production Operations Symposium, Oklahoma City, OK.

[10] Meyet, M., Dutta, R., Burns, C., Hughes, B., 2013 , "Comparison of Decline Curve Analysis Methods with Analytical Models in Unconventional Plays",pp.1-2, SPE 166365, New Orleans, Louisiana, USA.

[11] Shirman, E., 1998, "Universal Approach to the Decline Curve Analysis", pp.1-2, presented at the $49^{\text {th }}$ Annual Technical Meeting of The Petroleum Society in Calgary, Alberta Louisiana State University, Alberta, Canada.

[12] Hornik K, Stinchcombe M, White H., 1990, "Universal approximation of an unknown mapping and its derivatives using multilayer feed forward networks". Neural Netw 3(5):551-560.

[13] Ganesan, T., Elamvazuthi, I., Vasant, P., 2011, "Solving engineering optimization problems with the KarushKuhn-Tucker Hopfield neural networks", International Review of Mechanical Engineering 5 1333-1339.

[14] Brown M., Harris C., 1994, "Neural fuzzy adaptive modeling and control. Prentice-Hall, Englewood Cliffs. 